Return to the Manage Active Submissions page at http://spie.org/submissions/tasks.aspx and approve or disapprove this submission. Your manuscript will not be published without this approval. Please contact author_help@spie.org with any questions or concerns.

\title{
Observation of a rainbow of visible colors in a near infrared cascaded Raman fiber laser and its novel application as a diagnostic tool for length resolved spectral analysis Santosh Aparanji ${ }^{\mathrm{a}}$, V. Balaswamy ${ }^{\mathrm{a}}$, S. Arun ${ }^{\mathrm{a}}$, V. R. Supradeepa*a \\ ${ }^{a}$ Centre for Nano Science and Engineering, Indian Institute of Science, Mathikere, Bangalore, Karnataka, India 560012
}

\begin{abstract}
In this work, we report and analyse the surprising observation of a rainbow of visible colors, spanning $390 \mathrm{~nm}$ to $620 \mathrm{~nm}$, in silica-based, Near Infrared, continuous-wave, cascaded Raman fiber lasers. The cascaded Raman laser is pumped at $1117 \mathrm{~nm}$ at around $200 \mathrm{~W}$ and at full power we obtain $\sim 100 \mathrm{~W}$ at $1480 \mathrm{~nm}$. With increasing pump power at $1117 \mathrm{~nm}$, the fiber constituting the Raman laser glows in various hues along its length. From spectroscopic analysis of the emitted visible light, it was identified to be harmonic and sum-frequency components of various locally propagating wavelength components. In addition to third harmonic components, surprisingly, even $2^{\text {nd }}$ harmonic components were observed. Despite being a continuous-wave laser, we expect the phase-matching occurring between the core-propagating NIR light with the cladding-propagating visible wavelengths and the intensity fluctuations characteristic of Raman lasers to have played a major role in generation of visible light. In addition, this surprising generation of visible light provides us a powerful non-contact method to deduce the spectrum of light propagating in the fiber. Using static images of the fiber captured by a standard visible camera such as a DSLR, we demonstrate novel, image-processing based techniques to deduce the wavelength component propagating in the fiber at any given spatial location. This provides a powerful diagnostic tool for both length and power resolved spectral analysis in Raman fiber lasers. This helps accurate prediction of the optimal length of fiber required for complete and efficient conversion to a given Stokes wavelength.
\end{abstract}

Keywords: Lasers; Lasers, fiber; Lasers, ytterbium; Scattering, Raman; Lasers, Raman; Nonlinear wave mixing; Spectrum analysis; Harmonic generation and mixing; Image analysis; Visible lasers.

\section{INTRODUCTION}

Rare-earth doped fiber lasers have gained tremendous scope in the last decade due to their excellent power scalability and better thermal management ${ }^{1}$. However rare-earth doped fiber lasers provide power scaling in only specific wavelength bands with lots of white spaces in between. Raman fiber lasers are currently the only feasible and attractive solution for wavelength agility, and several architectures have been proposed to realise them ${ }^{2-4}$. The phenomenon of visible light generation in single-mode optical fibers operating in the high peak-power pulsed regimes has previously been reported, and explained to be through harmonic conversion and four-wave-mixing ${ }^{5-7}$. In this work, we report and analyze the rather surprising observation of a rainbow of visible colors, spanning $390 \mathrm{~nm}$ to $620 \mathrm{~nm}$, seen in the cladding of a silica-based, near infrared, continuous-wave, cascaded Raman fiber laser. These Raman fiber lasers essentially consist of a passive fiber with small effective area to increase the Raman gain coefficient in nested cavities separated by Stokes shifts. In many such architectures that realise a cascaded Raman fiber laser, the efficiency of the laser is well below the quantum limited efficiency between the pump and the desired final wavelength ${ }^{2}$. One of the reasons for this is the lack of proper termination of the cascaded Raman conversion. Though Raman filter fibers have been recently used in cascaded Raman lasers to achieve this goal, if the length of the filter fiber used is not optimal, it can lead to leakage of excess power in the cladding modes thereby reducing the efficiency of conversion. However, there does not exist any non-destructive, non-contact approach by which one can spatially resolve the power or the wavelength propagating locally within the core of the fiber. In order to determine what wavelength propagates at a given spatial location of the fiber, one would need to cleave the fiber at that location to connect to a spectrometer. However, our surprising observation of the visible rainbow in the cladding of the fiber can provide us with a powerful non-contact diagnostic tool to determine the locally propagating NIR core wavelengths at every spatial location of the fiber. We have developed a 
Return to the Manage Active Submissions page at http://spie.org/submissions/tasks.aspx and approve or disapprove this submission. Your manuscript will not be published without this approval. Please contact author_help@spie.org with any questions or concerns.

novel image-processing based algorithm that can take a static DSLR snapshot of the glowing Raman fiber and provide both length and power-resolved spectral analysis of the locally propagating NIR core wavelengths in the fiber. All this can be done "at a distance" without damaging the fiber in the process. This powerful diagnostic tool helps in optimizing the length of the Raman filter fiber required for complete conversion to a given Stokes wavelength and would pave the way for achieving quantum-limited efficiency in cascaded Raman lasers.

\section{EXPERIMENTAL SETUP}

Fig. 1 shows the schematic of an efficient and simplified Raman conversion module pumped at $1117 \mathrm{~nm}$ and providing an output at $1480 \mathrm{~nm}$ at full power ${ }^{3}$. At full power of $193 \mathrm{~W}$ from the $1117 \mathrm{~nm}$ pump, this Raman converter module provides $>90 \mathrm{~W}$ at the final Stokes wavelength of $1480 \mathrm{~nm}$. The Raman filter fiber provides a distributed loss beyond $1500 \mathrm{~nm}$ through the intrinsic cutoff of the fundamental mode, and ensures the termination of the cascaded Raman conversion at $1480 \mathrm{~nm}$.

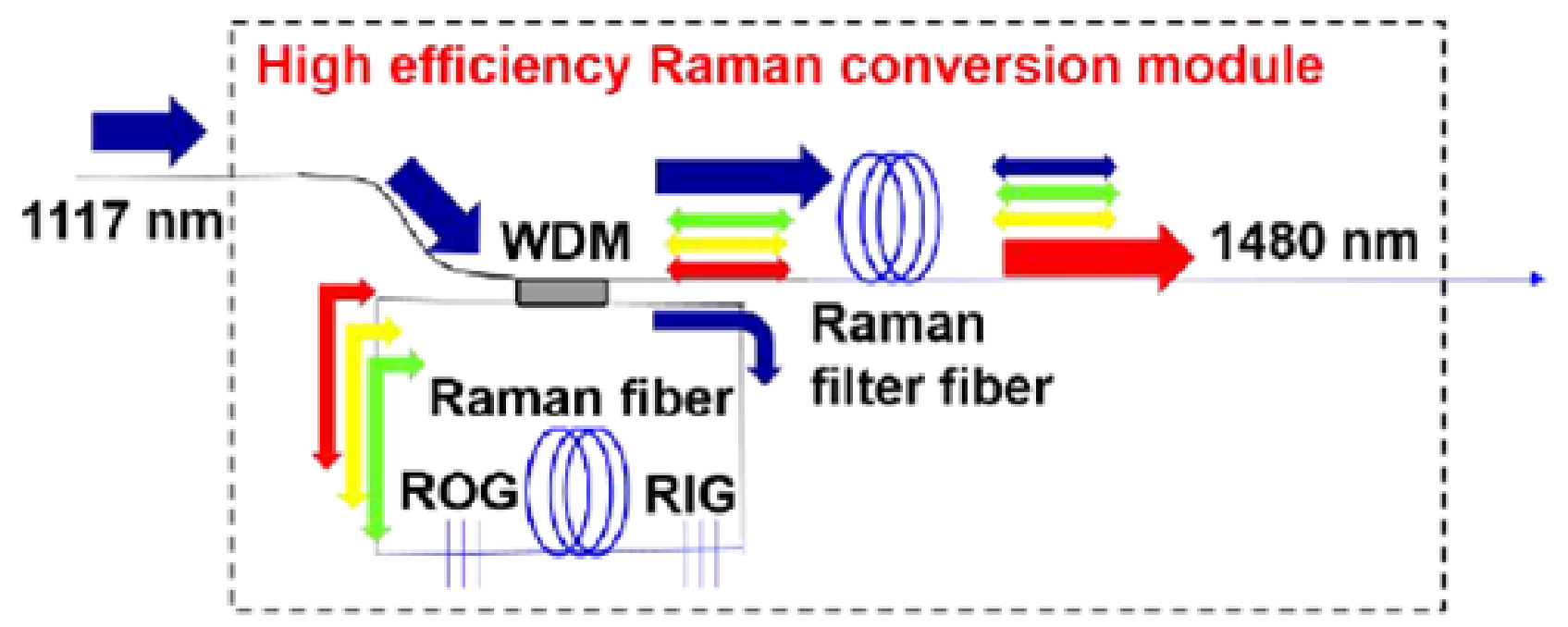

Figure 1. Schematic of an efficient cascaded Raman fiber laser pumped at $1117 \mathrm{~nm}$ and providing output at $1480 \mathrm{~nm}$.

With increasing pump powers of the $1117 \mathrm{~nm}$ laser, the cladding of the Raman filter fiber takes on a rainbow of visible colors and this is captured through a standard visible light camera such as a DSLR, and is shown in Fig. 2. This glow from the cladding of the fiber can be attributed to the scatter of the visible light through the high-index coating typical of a Raman filter fiber. From the figure it can be seen that this visible rainbow starts from the top of the spool (the input end of the Raman fiber) in a greenish hue, and with increasing length of the fiber the colors transition to yellow, light pink and a deep pinkish blue hue at the middle of the fiber spool. It appears that the fiber is emitting at longer wavelengths, going from green to a pinkish-red. However, strangely, this trend is abruptly terminated midway in the top fiber spool after the pinkish glow of the fiber, and the fiber starts glowing a deep blue hue. Thereafter with increasing length, the fiber glows at longer wavelengths, emitting light blue and cyan wavelengths all the way till the end of the Raman filter fiber. To determine the nature of the visible light emitted in the cladding, a hand-held visible-light spectrometer was used at different spatial locations close to the cladding of the fiber, and the spectra at different spatial locations of the fiber were recorded. 


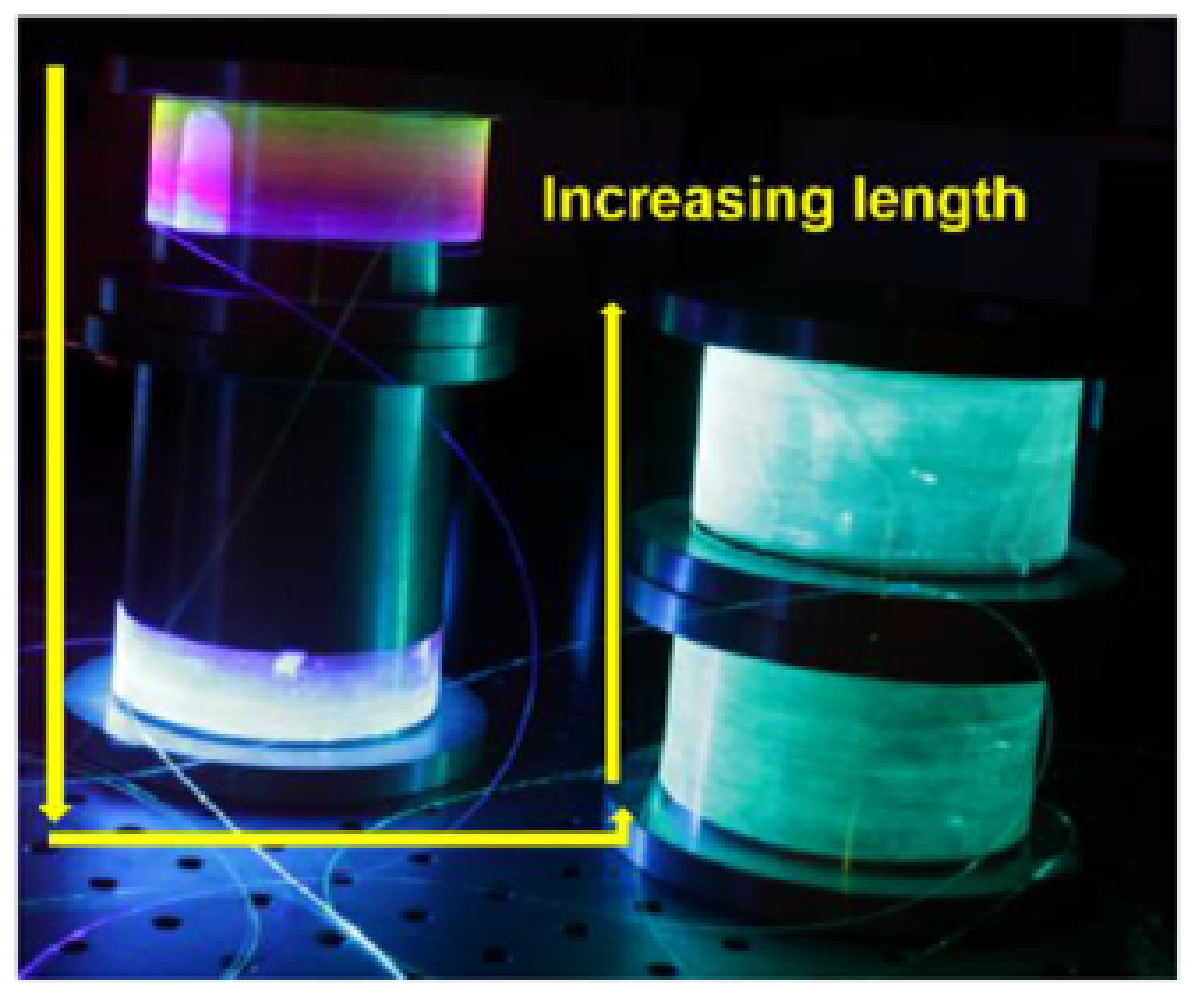

Figure 2. A static DSLR camera snapshot of the Raman fiber spools taking on a visible rainbow of colors in the cladding.

\section{RESULTS AND ANALYSIS}

(a)

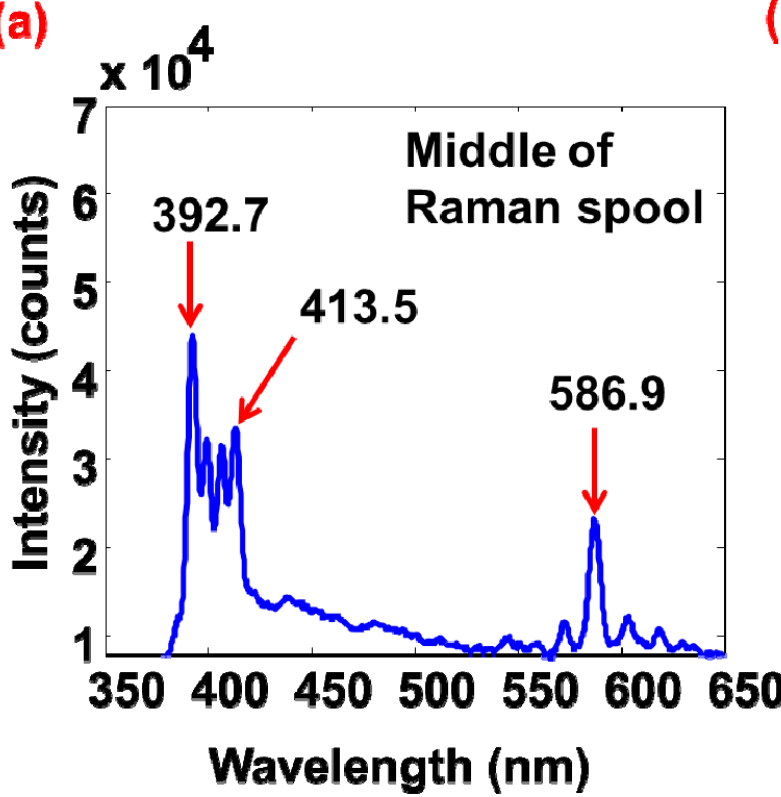

(b)

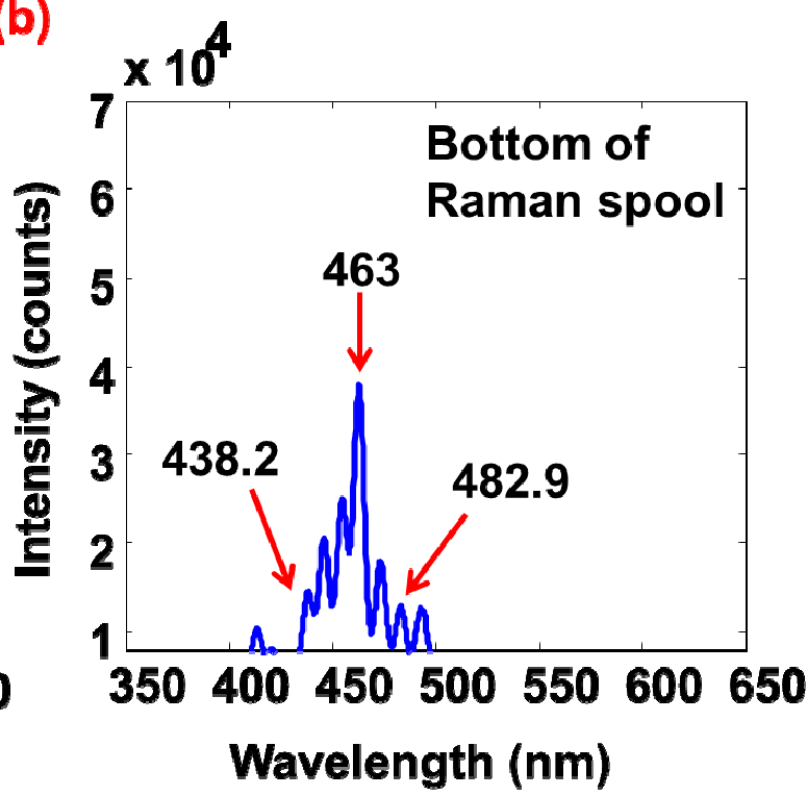

Figure 3. Representative visible spectrometer plots (background subtracted) taken close to the cladding (a) near the middle of the Raman fiber spool, and; (b) near the bottom of the Raman fiber spool. DB Check, Prod Check, Notes: 
Return to the Manage Active Submissions page at http://spie.org/submissions/tasks.aspx and approve or disapprove this submission. Your manuscript will not be published without this approval. Please contact author_help@spie.org with any questions or concerns.

Fig. 3 shows the representative visible spectrometer plots taken at two different spatial locations of the Raman filter fiber spool. Fig. 3(a) shows the prominent visible spectral components seen near the middle of the Raman fiber spool where the fiber is glowing a yellow and pinkish hues. Fig. 3(b) shows the dominant spectral components at the bottom of the fiber spool where the fiber is visibly glowing a bluish hue. Both these plots indicate the appropriate visible wavelength components at that location, but they also show something more interesting. They indicate that this visible rainbow does not continuously span all wavelengths but instead consist of distinct and sharp peaks at specific visible wavelengths. This indicates that this visible light generation is due to the harmonic conversion of the pump wavelengths and the various locally propagating Stokes wavelengths in the fiber, because of the innately discrete nature of the harmonic conversion process. Specifically, we hypothesize that the colors in the beginning of the Raman fiber that glow from green to the pinkish-red hue, and spanning wavelengths from $550 \mathrm{~nm}$ to $620 \mathrm{~nm}$, are due to the second harmonic generation of the pump wavelength at $1117 \mathrm{~nm}$ and its immediate higher order Stokes propagating in the Raman fiber. And the deep bluish hues from the middle of the spool to the cyan hues at the end of the fiber, spanning wavelengths from $390 \mathrm{~nm}$ to $490 \mathrm{~nm}$, are due to the third harmonic generation of the higher order Stokes propagating within the Raman filter fiber. Although it might initially appear strange that a silica fiber should exhibit second harmonic generation, it being a centro-symmetric material, this phenomenon of second harmonic generation in silica fibers has previously been reported and explained to be due to the anomalies in the Si-O structural rings ${ }^{8,9}$. A plot illustrating this visible light generation due to second harmonic generation and third harmonic generation as a function of length of the Raman fiber is shown in Fig. 4.

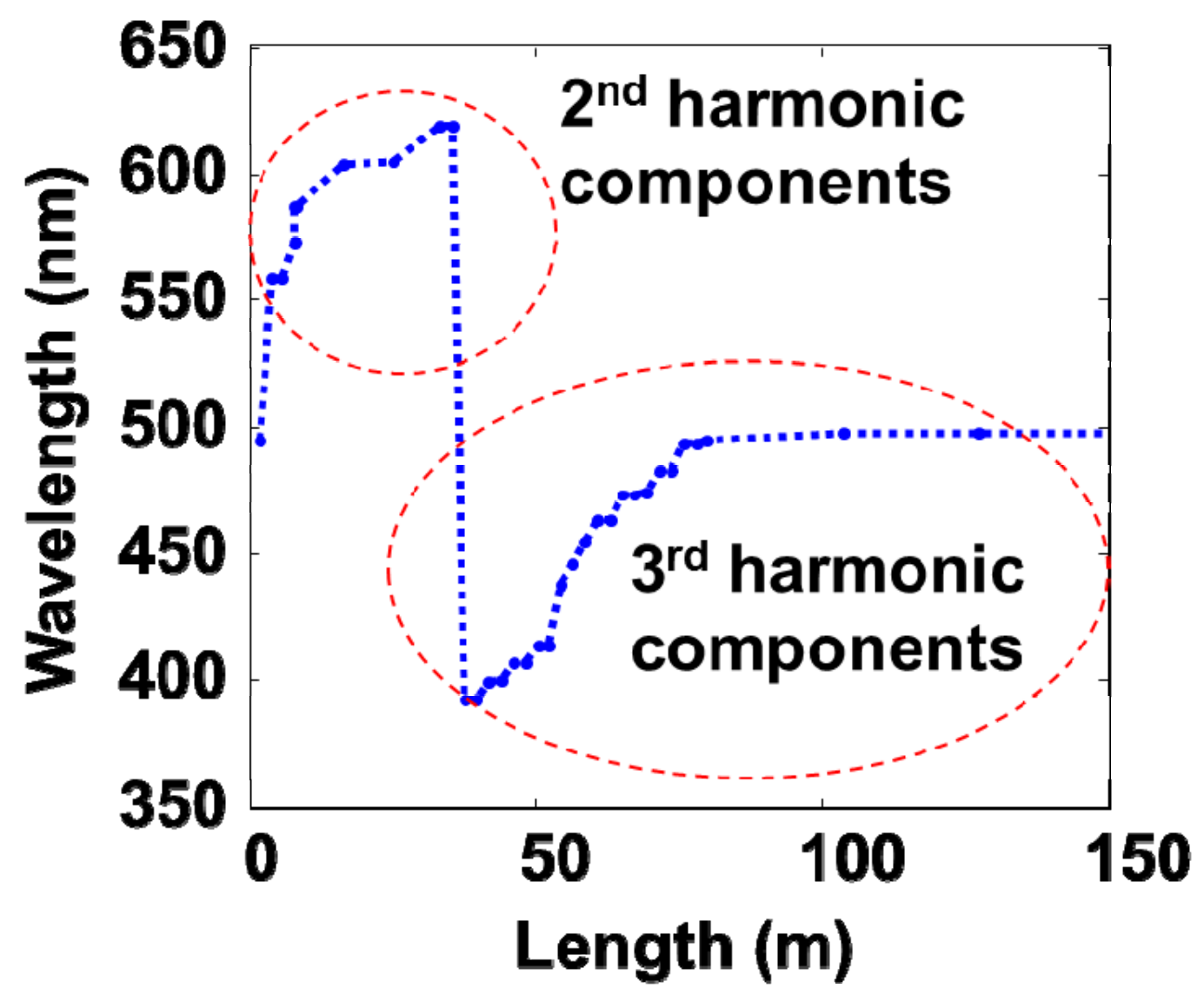

Figure 4. Length-resolved plot of the visible wavelengths emitted along the Raman fiber.

Because the visible light generation in the cladding of the fiber is a harmonic conversion process of the local corepropagating NIR Stokes wavelengths, in principle, one should be able to deduce the underlying Stokes wavelengths 
Return to the Manage Active Submissions page at http://spie.org/submissions/tasks.aspx and approve or disapprove this submission. Your manuscript will not be published without this approval. Please contact author_help@spie.org with any questions or concerns.

propagating in the core of the fiber merely by looking at the color emitted at that spatial location in the cladding. This is what we precisely did by developing a novel image-processing based algorithm that takes as an input a static DSLR snapshot of the glowing Raman fiber spool and produces as the output a length-resolved mapping of the various locally propagating Stokes wavelengths in the core of the fiber. In this algorithm, we determine the hue of a given pixel of the fiber cladding from its Red, Green, Blue (RGB) values in order to determine the exact wavelength at that spatial location. The hue of a given pixel is determined by converting the RGB pixels into the Hue, Saturation, Value (HSV) model, in order to decouple the hue from the intensity and degree of saturation of that pixel. Once the hue values are obtained, the visible light wavelength corresponding to that pixel can be estimated by using a Munsell color wheel ${ }^{10}$. In this way, every pixel of the image that corresponds to the glowing cladding of the fiber can be assigned an effective wavelength in the visible spectrum. Thereafter, the wavelengths up to the pinkish hue of the fiber are doubled to account for the second harmonic generation, and the estimated visible wavelengths from the deep blue hues of the fiber are tripled to account for the third harmonic generation. This distinction between the second harmonic and the third harmonic region can be easily made by using an image segmentation algorithm. As the input power of the $1117 \mathrm{~nm}$ pump changes, the hues in the rainbow are visibly different, which can be easily captured by a DSLR camera and analysed by the novel image-processing algorithm. This would give us both a power-resolved and a length-resolved picture of the effective center Stokes wavelengths propagating in the core of the fiber.

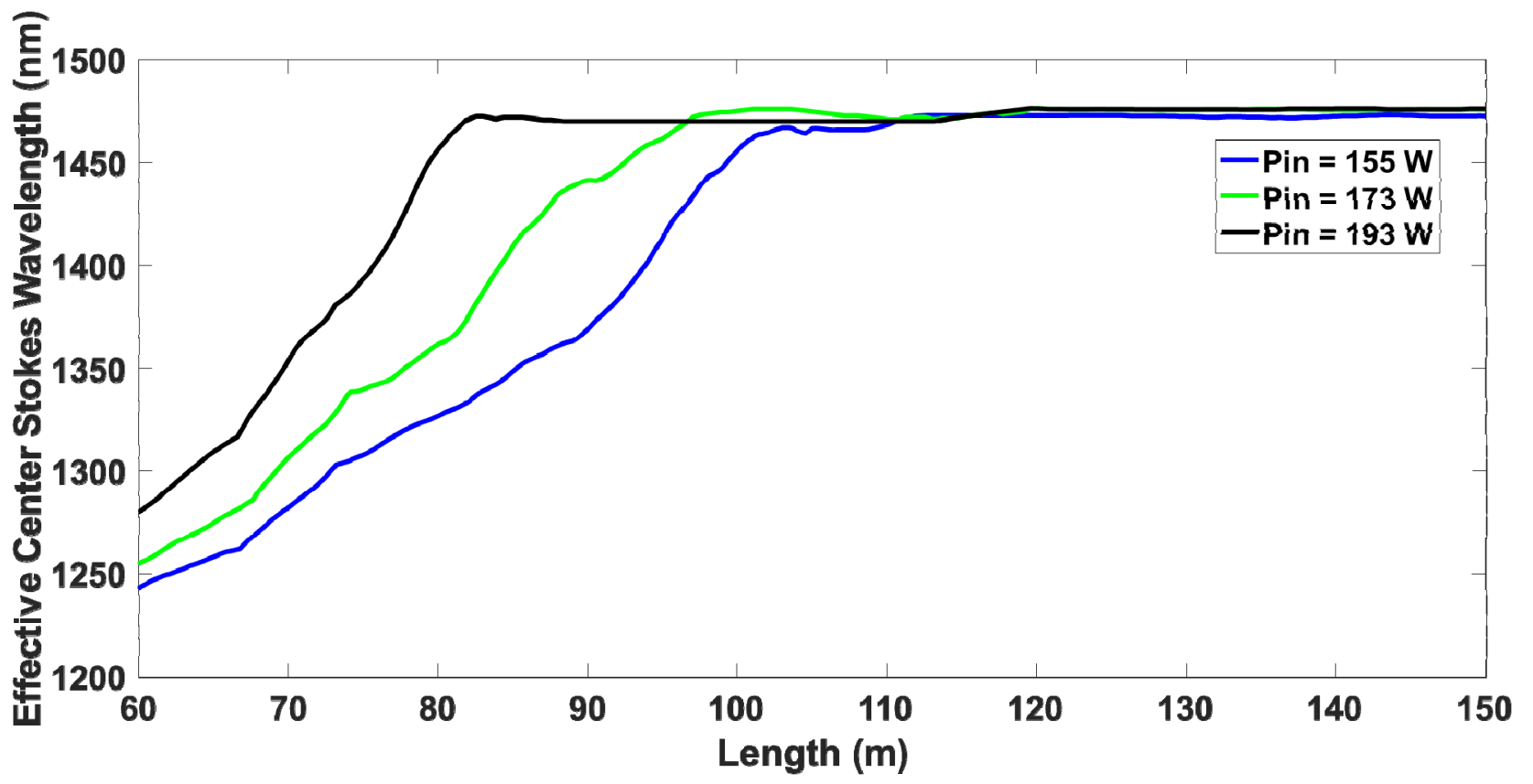

Figure 5. Length-resolved plot of the effective center Stokes wavelength propagating in the third harmonic region of the fiber.

Fig. 5 shows the power-resolved and length-resolved plot of the effective center Stokes wavelength propagating in the core of the fiber where the colors emitted from the cladding of the fiber are due to the third harmonic generation. Because the Stokes wavelengths propagate in the core at discrete steps (which for $1117 \mathrm{~nm}$ pump correspond to steps at $1175 \mathrm{~nm}, 1240 \mathrm{~nm}, 1310 \mathrm{~nm}, 1390 \mathrm{~nm}$ and $1480 \mathrm{~nm}$ ), this plot and the image-processing based analysis cannot be considered as fully accurate. This is why the prediction of the locally propagating Stokes wavelength from this imageprocessing based analysis is merely an effective center Stokes wavelength, which assumes that only a single Stokes wavelength in the core undergoes third harmonic or second harmonic conversion to give rise to a color. But this crude assumption is sufficient to predict to a certain degree of accuracy the length of the Raman fiber required for the complete conversion to a given Stokes wavelength.

From Fig. 5, it can be seen that for a given input pump power, greater length of the Raman fiber used would lead to a higher order Stokes conversion. However, because of the use of the Raman filter fiber, this conversion stops beyond $1480 \mathrm{~nm}$, which is succinctly captured in the plot. The plot also shows that with increasing input pump powers, a shorter 


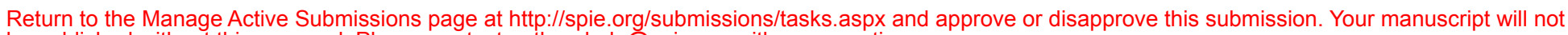
be published without this approval. Please contact author_help@spie.org with any questions or concerns.

length of the Raman filter fiber is sufficient to convert to a given Stokes wavelength. Specifically, only around $80 \mathrm{~m}$ of Raman filter fiber is required in order to convert $193 \mathrm{~W}$ of input pump power at $1117 \mathrm{~nm}$ to the final Stokes of $1480 \mathrm{~nm}$. However, at a lower input pump power of $155 \mathrm{~W}$, approximately $110 \mathrm{~m}$ of the fiber would be required to achieve full conversion to $1480 \mathrm{~nm}$.

In summary, in this work, we have reported and analyzed the surprising observation of a visible light rainbow in a NIR, continuous-wave cascaded Raman fiber laser. We identified the visible light generation to be due to second and third harmonic conversion of the pump and the locally propagating Stokes wavelengths within the core of the fiber. A novel image-processing based algorithm was developed as a non-contact, non-destructive approach to estimate the locally propagating Stokes wavelengths within the core of the fiber "at a distance". This would pave the way for optimizing the length of Raman fiber used for complete conversion to a given Stokes wavelength and help in improving the efficiency of cascaded Raman lasers.

\section{REFERENCES}

[1] Richardson, D. J., Nilsson, J. and Clarkson, W. A., "High power fiber lasers: current status and future perspectives [Invited]," J. Opt. Soc. Am. B 27, B63-B92 (2010).

[2] Supradeepa, V. R., Feng, Y. and Nicholson, J. W., "Raman fiber lasers,” J. Opt. 19.2, 023001 (2017).

[3] Supradeepa, V. R., Balaswamy, V., Arun, S. and Chayran, G., "A Simplified Architecture for High Efficiency Cascaded Raman Fiber Lasers," in Conference on Lasers and Electro-Optics, OSA Technical Digest, paper SM2Q.5 (2016).

[4] Arun, S., Balaswamy, V., Aparanji, S. and Supradeepa, V. R., "High Power, Grating-Free, Cascaded Raman Fiber Lasers" Conference on Lasers and Electro-optics (CLEO) Europe, CJ-2.4, (2017).

[5] Gomes, A. S. L., Osterberg, U. and Taylor, J. R., "Spectral and Temporal Investigations of Nonlinearities in a NonPolarization Preserving Single-Mode Optical Fibre,” Appl. Phys. B 41, 235-240 (1986).

[6] Osborne, R., "Near-infrared continuum generation with upconversion into the visible in SiO2 single-mode fiber," Opt. Lett. 19, 1955-1957 (1994).

[7] Jauregui, C., Steinmetz, A., Limpert, J. and Tunnermann, A., "High-power efficient generation of visible and midinfrared radiation exploiting four-wave-mixing in optical fibers," Opt. Express 20, 24957-24965 (2012).

[8] Gabriagues, J. M. and Fevrier, H., "Analysis of frequency-doubling processes in optical fibers using Raman spectroscopy," Opt. Lett. 12, 720-722 (1987).

[9] Osterberg, U. and Margulis, W., "Dye laser pumped by Nd:YAG laser pulses frequency doubled in a glass optical fiber," Opt. Lett. 11, 516-518 (1986).

[10] Nickerson, D., "History of the Munsell color system and its scientific application." JOSA 30.12: 575-586 (1940). 